\title{
Aplicação da metodologia lean seis sigma no desenvolvimento de um programa de gestão da inovação, no Hospital Santa Izabel
}

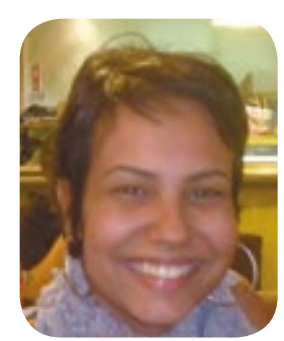

Taiana Barbosa de Freitas Galderice

RESUMO

Este artigo visa compreender os resultados da implementação de programa de gestão da inovação em um hospital filantrópico de grande porte, localizado na região Nordeste do Brasil, aplicando a ferramenta de gestão empresarial Lean Seis Sigma (LSS) no gerenclamento de projetos aplicados a dinâmica operacional administraiva assistizial, excluindo fatores clínicos do paciente. Foi realizada uma pesquisa explicativa sobre a metodologia Lean Seis Sigma para melhoria do processo de gestáo da inovaçao instlucional, considerando ferame corespondente (DMAIC - Definir, Medir, Analisar, Implementar e Controlar) como forma de identicar, analisar e implementar soluçóes. Neste estudo verilicou-se que as soluçóes de inovações aplicadas a partir de projetos LSS permitiram methor mo no processo de gestáo hospitalar, constiluindo um modelo que condiciona um pensamento eco com soluções inovadoras efetivas.

DESCRITORES: projetos; Gestão da inovação Lean Seis Sigma; Inovação em saúde; Lean Healthcare.

MÉTODO

Como método de análise, foi utilizado o estudo de caso qualitativo explicativo, empregado até os dias de hoje nas ciências sociais e na pesquisa qualitativa organizacional. A partir de uma analise qualitativa dos fatos e fatores determinantes, esse estudo de casos foi estruturado da seguinte forma: (a) revisão bibliográfica; (b) relato do caso escolhido; (c) resultados conquistados; e (d) anâlise preliminar qualitativa da aderência das soluç̃os implementadas.

1. INTRODUÇÃO

As organizações estão sempre em busca de estratégias que as impulsionem a uma posição de destaque,
garantindo-Ihes maior competitividade no mercado. $\mathrm{Na}$ área hospitalar, a busca por novas técnicas e práticas de gestão está possibilitando conquistas significativas em um cenário de crise econômica para este segmenando-as cada vez mais eficientes e eficazes.

Para que obtenham bons resultados, as organizações adotam ferramentas e metodologias que impactam diretamente no desempenho dos seus processos e de seus colaboradores, gerando mudanças, novas metas e novos modelos de performance, de acordo com a necessidade do cliente.

Esforços para as inovações de forma a enfrentar a concorrência, deve considerar a participação de todo - colaboradores, fornecedores e clientes -, o que e fundamental para o sucesso da empresa, pois são as pessoas que all trabalham e formam a cadeia de valor que são as detentoras das habilidades e conhecimentos para a execuçáo dos produtos, processos ou

$O$ interesse em realizar este artigo surge na necessidade de identificar como um hospital filantrópico, que já possui como principal característica a escassez de recurso financeiro, estaria inovando nos processos de gestão organizacional e de projetos, devido à crescente e constante importância das inovações exigidas pelo mercado de saúde. Acredita-se que a relevância deste trabalho está pautada na contribuição para idenificcação das praticas de gestão que contribuam para a agregação de valor aos serviços administrativos e assistenciais prestados, por intermedio de equipes bem treinadas, padronização de processos e redução de variabilidade nas entregas. Nesse sentido, realizou-se o estudo de um caso qualitativo explicativo. A partir de uma análise qualitativa dos fatos e fatores determinantes, esse estudo de casos foi estruturado da seguine forma: (a) revisão bibliográfica; (b) relato do caso preliminar qualitativa da aderência das soluções impreliminar qualita
plementadas.

\section{REVISÃO BIBLIOGRÁFICA}

2.1. Programa de Gestão da Inovação

A baixa adesão e morosidade da área da saúde em aderir à modernização da gestão empresarial e tecnológica é reconhecida e evidente. Uma das causas levantadas é o temor dos gestores de que a administração estruturada na inovação de projeto implique em interferência na conduta clínica ou até mesmo nas condições inerentes ao cliente paciente. É importante entender essa cultura no Brasil dentro das condições materiais em que está sustentada a gestão hospitalar filantrópica, na qual atua na busca vital e prioritária de recurso financeiro com origem predominante na iniciativa governamental e na sociedade.

O entendimento e a absorção do conceito de gestão da inovação têm chegado ao âmbito hospitalar de maneira tímida nos últimos anos, apesar de ser um nicho de mercado extremamente competitivo, em que processos e produtos que agregam valor ao cliente paciente ou cliente médico garantem diferencial no mercado e fidelização deste público. A busca de diferenciais e a sustentabilidade, das posições de mercado e financeira, têm sido discutidas, sobretudo, diante de um contexto que se apresenta marcado pelo acirramento da concorrência e por constantes mudanças na dinâmica produtiva nacional, principalmente a velocidade da tecnologia clínica no âmbito hospitalar.

A necessidade de estabelecer patamares mais elevados de desempenho e competitividade sustentáve estimula a reflexão acerca dos fatores que afetam a performance, direcionando ao pensamento de inovação. A gestão da inovação nas organizações vem ganhando espaço recentemente, tratando a inovação de forma responsavel, a partir de estrategia, modelos, recursos e ferramentas. Em paralelo, busca mudar uma visão equivocada de ser algo desorganizado e não sistemático, facilitando e mudando a maneira como as pessoas lidam com a resoluçáo de problemas com a apresentação de produtos e soluçoes eficientes. A gestão com foco na inovação está muito ligada à mudança da cultura no ambiente institucional, mas também pode envolver a aplicação da tecnologia tanto nos processos criativos quanto no desenvolvimento de produtos e serviços.

No segmento da indústria é possível identificar alguns modelos de gestão da inovação sendo praticados há algumas décadas, como o caso Toyota:
A empresa tem desbancado montadoras americanas há tempos e os motivos, muitas vezes, são menos óbvios do que parecem. Mesmo quando as companhias americanas alcançam os japoneses em faturamento, estes apresentam uma margem de lucro muito maior. Uma das explicações é que a Toyota implementa melhorias de produtos, processos e falhas em uma velocidade absurda. E isso tudo está ligado à maneira como a organização encara essa inovação. Enquanto as companhias americanas deixam a inovação e a melhoria dos processos nas mãos somente de alguns funcionários ou gestores mais especializados, há muito tempo a Toyota acredita que todos os empregados podem ser 'resolvedores' de problemas, criadores e agentes de mudança. Na prática, a Toyota deu a cada empregado as habilidades, as ferramentas e a permissão para resolver problemas à medida que eles surgiam - e também para afastar novos problemas antes que eles ocorram.

Agestão da inovação é responsável por propiciar um terreno fértil, onde barreiras para o surgimento, implementação e execução de ideias são mitigadas a parti de tecnicas e ferramentas que estruturam pensamento sem bloqueio de ideias: identificando o problema ou oportunidade, compreendendo o comportamento da situação e/ou daquele que sofre com o problema, com levantamento e estudos de evidencias, levantamento de propostas (ideias) que neutralizem o problema e estruturação e execução de ações.

Segundo o dicionário, inovação está traduzido como "aquilo que constitui algo novo" ou ainda "desenvolvimento de novos produtos, metodos ou conceitos", trazidas como definiçóes mais comuns e populares para descrever processos de inovação. Mas, como dito anteiormente, existem ferramentas necessárias que apoiam a identificação e/ou construção de algo inovador.

2.2. Ferramentas de gestão: Lean Seis Sigma MBOK® e Balance Scorecard

Seis Sigma é um conjunto de práticas que busca dologia estruturada, focada na excelência e competividade, por meio da melhoria continua dos processos de produção de um bem ou serviço, levando em conta todos os aspectos importantes de um negócio. A metodologia Lean, com origem no Sistema Toyota de Produção, busca eliminar desperdícios, ou seja, excluir tudo o que não agrega valor para o cliente definir uma nova cadência nas atividades e eficiência à empresa. 
Tabela 1. O Lean e o Seis Sigma

\begin{tabular}{|c|c|c|}
\hline & LEAN & SEIS SIGMA \\
\hline $\begin{array}{l}\text { Relação de } \\
\text { causa e efeito }\end{array}$ & $\begin{array}{l}\text { Melhorar a cadeia de } \\
\text { valor com redução do } \\
\text { desperdício. }\end{array}$ & $\begin{array}{l}\text { Melhorar o processo } \\
\text { com redução } \\
\text { da variabilidade } \\
\text { (defeitos). }\end{array}$ \\
\hline $\begin{array}{c}\text { Teoria } \\
\text { principal } \\
\text { de cada } \\
\text { metodologia }\end{array}$ & 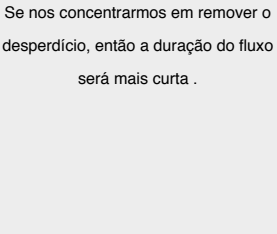 & $\begin{array}{l}\text { Se nos } \\
\text { concentrarmos em } \\
\text { reduzir a variação, } \\
\text { então teremos } \\
\text { resultados de } \\
\text { processo mais }^{\text {aniformes. }}{ }^{3}\end{array}$ \\
\hline
\end{tabular}

\section{1.htpp:///porta
-empresas}

2. NAVE D. Como comparar o Seis Sigma, o Lean e a Teoria das Restriçês. 3. NAVE D. Como comparar o Seis Sigma, o Lean e a Teoria das Restriçöes.

Ao integrar as duas metodologias, possibilita que a empresa usufrua dos pontos fortes de ambas estratégias. O programa resultante dessa integração é denominado Lean Seis Sigma (LSS) e constitui uma estratégia abrangente de inovação e eficaz para a solução de problemas e também para a criação de novos processos e produtos. A relação entre processos e projetos está na organização das atividades envolvidas e desenvolvidas pelos times de projetos. As empresas buscam a execução de projeto quando essa organização identifica oportunidades de melhorias além do seu limite operacional, situações normalmente provocadas por influências internas e/ou externas que geram algum mpacto na rentabilidade operacional e/ou financeira.

No âmbito de projetos, na década de 1960, duas instituições sem fins lucrativos foram criadas para padronizar o gerenciamento de projetos e avançar no estado da arte nessa área do conhecimento: o Institute for Project Management Association (IPMA), em 1965, na Europa sendo a primeira organização internacional criada com esse foco; e o Project Management Institute (PMI), em 1969, nos Estados Unidos. O PMI se dedica ao avanço no gerenciamento de projetos, promovendo atividades, estudos, eventos, treinamentos e bibliografia especializada nessa área do conhecimento, sendo o PMBOK ${ }^{\circledR}$ sua publicação elaborada para padronizar e divulgar as boas práticas em gerenciamento de projetos.

O gerenciamento de projetos e a aplicação de conhecimentos, habilidades, ferramentas e técnicas às atividades do projeto, a fim de atender seus requisitos, bem como dos stakeholders. São extremamente importan- tes à adaptação das diversas ferramentas as diferente necessidades de cada projeto, adequando à complex dade de cada um. O PMBOK® descreve 42 processos em 5 grupos (Iniciação; Planejamento; Execução; Monitoramento e Controle, e Encerramento), dividindo tais processos em 9 areas de conhecimento (integração, escopo, tempo, custo, qualidade, recursos, comunicação, risco e aquisiçōes). Cabe à equipe do projeto aplicar os conhecimentos que julgar necessarios, de modo a se ajustar ao projeto especifico e à organização.

$O$ conceito de Balanced Score Card (BSC) foi lançado por David Norton e Robert Kaplan, em Harvard, na década de 90 . O BSC é um modelo de gestão estratégica que apoia a instituição na mensuração dos progressos, objetivos, indicadores e iniciativas institucionais. A mensuração considera quatro perspectivas estratégicas - Financeira, Mercado/Cliente, Processos Internos e Aprendizado. Com este desenho, o BSC se transformou em uma potente ferramenta para o planejamento estratégico empresarial, traduzindo missão e as estrategias das empresas em um conjunto de medidas de desempenho que apoia um sistema de medição gestão estratégica.

O uso dessas ferramentas de gestão foi introduzido inicialmente em setores como Indústria, Tecnologia de

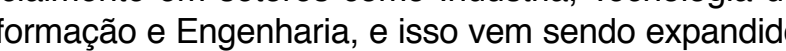
a outras áreas, como Terceiro Setor e Saúde.

\section{RELATO DO CASO SELECIONADO}

3.1. Breve descrição do caso

Em observância à tendência de gestão em saúde focada na excelência operacional, a partir de uma visão sistêmica com foco em resultados e segurança do paciente, o hospital de estudo iniciou o ano de 2014 potencializando caminhos em direção aos benefícios proporcionados pela execução de projetos operacionais interligados as planajamento estraleglco plunanual.

O hospital de estudo está classificado como filantrópico, ou seja, sem fins lucrativos, possui mais de 500 eitos, sendo 85 destes em unidades de terapia intensiva, elevando-o a categoria de hospital de grande porte, 16 salas de procedimentos cirúrgicos e hemodinâmicos e mais de 30 especialidades médicas com atendimento médio superior a 2.000 pacientes/dia.

Em um cenário com enfrentamentos importantes, potencializado por uma elevada competitividade e cenário econômico pouco favoravel às instituições de saúde, a união entre inovação e tradição foi um dos desafios enfrentados ao longo do processo. Neste contexto, a decisão de escolha por um modelo de gerenciamento por processos foi estratégico para o início de uma trans- formação organizacional direcionada à filosofia Lean Healthcare

Lean Healthcare é uma filosofia que organiza os processos hospitalares, por meio de um conjunto de conceitos e técnicas que definem valor sob a ótica do paciente, e reconhecido como um dos mais avançados modelos de gestão de hospitais do mundo, sendo largamente empregado em hospitais de países como E.U.A., Canadá, Inglaterra e Austrália.

A aplicabilidade das metodologias Lean (metodologia Toyota de Produção) e do Seis Sigma (metodologia Motorola e General Eletric) na área da saúde ainda é algo inovador, apesar de alguns hospitais das regiões Sul e Sudeste do Brasil já terem adotado há mais de uma década. Neste panorama, esta instituição hospitalar, que possui mais de 120 anos de existência, buscava no início de 2014 uma mudança cultural direcionada ao modelo mental, focado para processos enxutos, de fluxo contínuo e redução de variabilidade dos métodos de trabalho, ou seja, um modelo de gestão sustentável, dos processos hospitalares.

Onto operacionais escolhido pelo hospital estudado tem como objetivos institucionais garantir eficiência operacional, melhoria do atendimento aos clientes e, simultaneamente, potencializar o controle de custos. Para isso foi necessário o envolvimento direto e patrocínio da alta gestão, bem como o engajamento da média gerência ao time operacional. A estratégia de implantação envolveu investimentos em capacitação e formação técnica, de colaboradores do nível estratégico, tatico e operacional, na metodologia Lean Seis Sigma, sendo este custo neutralizado já com os resultados dos primeiros projetos apresentados e finalizados.

A formação teve como objetivo capacitar colaboradores nos conhecimentos e fundamentos da metodologia LSS e na aplicação das suas principais ferramentas, de forma a habilitá-los a conhecer os fundamentos dos projetos de melhoria contínua, com o propósito de eliminar atividades que não agregam valor ao cliente, garantindo a segurança da assistencia prestada em equilibrio com a sustentabilidade financeira do hospita.

os níveis de formação foram:

- Yellow Belt - perfil predominantemente de membros da equipe tecnico-operacional (Técnicos de Enfermagem, Auxiliares e Assistentes Administrativos, Enfermeiros, Auxiliar de Suprimentos, Farmacêuticos, Médicos), capacitando-os a participar de projetos como membros do time e suporte técnico aos Green Belts.

Green Belts -perfil do grupo predominantemente

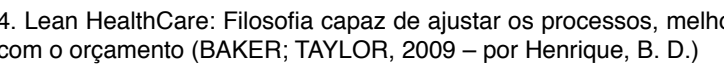

de coordenadores e gerentes, das áreas assistenciais e administrativas, com habilidade na utilização de conceitos e ferramentas de lógica, álgebra, raciocínio lógico e capacidade para utilizaçáo de software estatístico. Conhecimento consolidado de sua área funcional com capacidade de focar em processos críticos, bem como de administrar múltiplas atividades simultaneamente. Esses colaboradores foram habilitados a negociar visão e expectativas dos projetos.

- Black Belt - grupo formado pelos melhores participantes do Green Belt, com perfil para serem especialistas capacitados a liderar projetos complexos e prioritários, com foco no aumento da lucratividade e redução de desperdícios.

- Deployment Champion - grupo de executivos da organização, com visão de ganho de processos e ecoômicos, identificação de metodologias e resultados. A participação nesses cursos possibilitou aos colaboradores oportunidades únicas, pois, além do conhecimento técnico adquirido, também puderam vivenciar projetos no âmbito interdisciplinar, possibilitando troca de expeA disos sobre um mesmo problema. A diversidade das áreas promoveu debates, discussões e o nascimento de novos projetos. Análise criteriosa das competências e dos processos permite identificar os gaps de inovação e desenvolver um plano de ação para efetivamente inserir a gestão da inovação na organização.

Para formar um time focado na gestão de inovação utilizando metodologia LSS, desenvolver projetos e implementá-los nos primeiros dois anos, foi necessário monitoria de especialistas do mercado, garantindo a aplicabilidade da metodologia com segurança e obtenção de resultados rápidos. No primeiro ano de implementação (entre os anos 2015 e 2016) foram iniciados 53 projetos tocados em melhorias operacionais, financeiras e inovação de processos, que foram classificados e distribuídos de acordo com as perspectivas do Balancet Score Card (BSC), alinhados às categorias do planejamento estratégico do hospital:

Tabela 2. Classificação dos projetos LSS

Perspectiva do BSC $\quad \begin{gathered}\text { Categoria do Planej } \\ \text { Estratégico }\end{gathered}$

Cliente Estratégico Reconhecimento institucio

Processos Internos Redução de Custos Fonte MV - Módulo Estratégico 

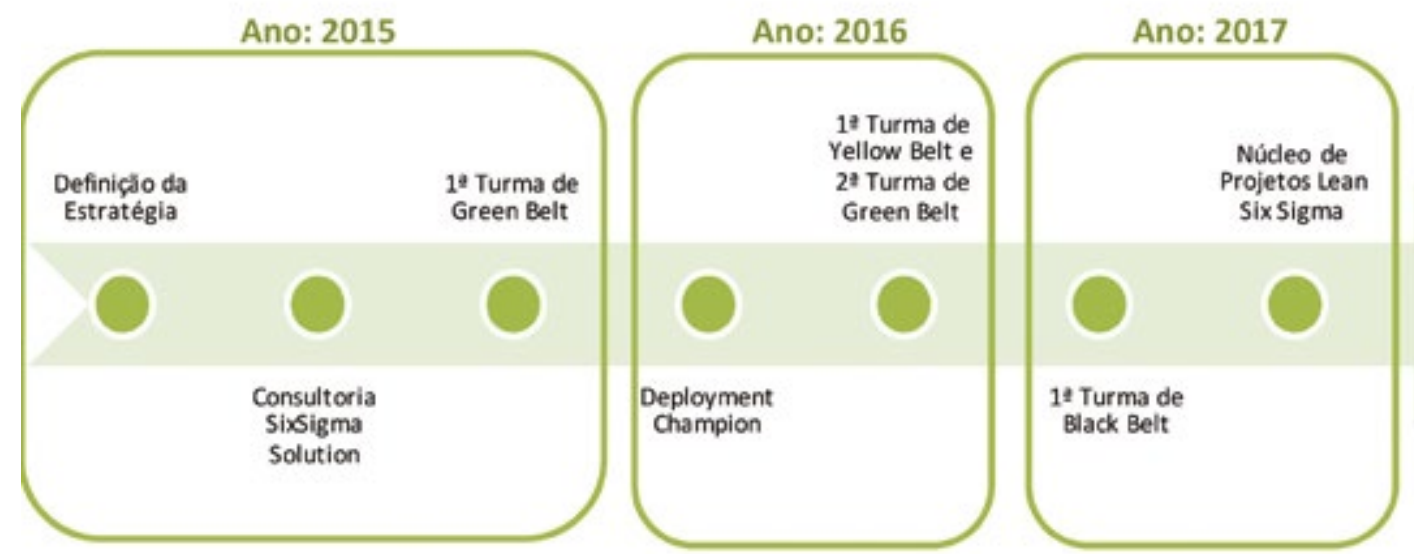

urada no hospital filantrópico de estudo, sendo que as soluções ocorreram em quatro instâncias igualmente mportantes: pessoas, processos, sistema (a ferramenta adquirida) e cultura organizacional.

4.1. Ganho de capital intelectual

Durante o projeto de implantação do LSS, foi constatada a ausência de um responsável para o gerenciamento da equipe de Belts. Na impossibilidade de contratação de um profissional em tempo integral à atividade de gestão de projetos, o hospital identificou um colaborador que foi direcionado para função exclusiva na gestão do pipe line de projetos. Essa atividade mostrou ter impacto favorável na efetividade no modelo de gestão de projetos e inovação, bem como número de
colaboradores formados na metodologia LSS.

4.2. Ganho Operacional e Financeiro

Todos os projetos LSS seguiram a metodologia DMAIC, na qual cada letra indica uma das cinco fases que a caracterizam: Definição, Medição, Análise, Implementacão das soluções e Controle do processo. Após o treinamanto institucional recebido, o líder de projeto ós o treinaa identificação do problema e a aprovacãa do pré-projeto. Deste universo de projetos, dezesseis formm finaDes é dezembro de 2016. Neste momento já foi lizados a dezer de projes reais, diretos e indiretos. Dentre eles, temos:

- Ganho médio de 10 minutos por atendimento na - Ganho médio de 10 minutos por atendimento na Recepcão do pronto atendimento até a clacsificaca da Recepçáo do pronto atendisto ale a Classificação de mento e reduzindo o tempo médio de permanencia no ambiente de emergência hospitalar. - Redução de $50 \%$ no tempo de leito bloqueado por
paciente necessidade de manutenção, possibilitando aumento

- Redução de 30\% no tempo de liberação do leito clínico pela Hotelaria.

Redução no tempo de liberação de leitos de terapia into cardiologia, otimizando da sala operadíaca e mitigando risco de suspensão cirúrgica.

- Melhoria no processo de dispensação da dieta para pacientes, inicialmente atendidos pelo Sistema Unico de Saude, expandido ponde - Inplan de in ternaçá do hospital.

co-obstrutivas no pronto atendimento pediatrico, gerando redução de custos com materiais e medicamentos Benefícios conquistados com a excuño de tos Lean Seis Sigma para:

- Hospital:

- Aumento de capacidade (nivelamento dos processos, melhorias operacionais).

- Redução de custos (redução de desperdícios) e maior rentabilidade.

- Inovações e processos mais ágeis.

- Melhor imagem perante a sociedade.

- Pacientes:

- Melhor qualidade (redução de esperas, movimentação e variabilidade).

Maior satisfação (fluxo contínuo, gestão visual).

- Maior segurança (fluxos e processos padronizados, redução de riscos).

- Colaboradores:

- Maior satisfação (visão sistêmica e maior envolvimento dos colaboradores)

Redução de stress (padronização de atividades e redução de burocracias)

- Aprendizado e desenvolvimento (treinamentos, capacitação, atividades práticas).

A neutralizacão de resistências e as dificuldades vivenciadas no ińcio de implantação deste modelo de verenciamento de projetos foram muito bem adminisTradas pela alta gestão do hospital, que impulsionou, 作

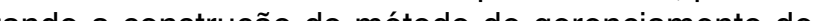
projetos baseado em uma filosofia sólida e focada na inovação.

\section{RESULTADOS CONQUISTADOS}

Mediante a implementação da filosofia Lean Healthcare, considerando integração entre as ferramentas LSS, BSC e PMBOK®, a gestão de inovação foi estru-
Tabela 3. Ganhos de Projetos

\begin{tabular}{|c|c|}
\hline Intervenção & Ganho \\
\hline $\begin{array}{l}\text { Otimização do tempo } \\
\text { de atendimento } \\
\text { da recepção até } \\
\text { a classificação de } \\
\text { risco na emergência } \\
\text { pediátrica }\end{array}$ & $\begin{array}{l}\text { Redução no tempo de atendimento } \\
10 \mathrm{~min} \text { por paciente }\end{array}$ \\
\hline $\begin{array}{l}\text { Redução do tempo de } \\
\text { liberação de leitos da } \\
\text { unidade de internação } \\
\text { para o setor de } \\
\text { regulação de leitos }\end{array}$ & Redução média de $77 \mathrm{~min}$ por leito \\
\hline $\begin{array}{l}\text { Redução do tempo para } \\
\text { início de hemodiálise }\end{array}$ & $\begin{array}{l}\text { Redução média de } 26 \mathrm{~min} \text { por } \\
\text { paciente }\end{array}$ \\
\hline $\begin{array}{l}\text { Redução do tempo } \\
\text { de liberação de leitos } \\
\text { de unidade de terapia } \\
\text { intensiva }\end{array}$ & $\begin{array}{c}\text { Redução média de } 3 \text { horas por } \\
\text { paciente }\end{array}$ \\
\hline $\begin{array}{l}\text { Redução do tempo } \\
\text { de bloqueio de leitos } \\
\text { por necessidade de } \\
\text { manutenção }\end{array}$ & Redução média de 5 dias por leito \\
\hline $\begin{array}{l}\text { Adequação nos } \\
\text { processos de terapia } \\
\text { medicamentosa na } \\
\text { clínica da dor }\end{array}$ & $\begin{array}{l}\text { Redução de custo anual de } 150 \text { mil } \\
\text { reais }\end{array}$ \\
\hline $\begin{array}{l}\text { Redução de múltiplas } \\
\text { punçoes periféricas em } \\
\text { pacientes pediátricos }\end{array}$ & $\begin{array}{l}\text { Redução de custo anual de } 40 \text { mil } \\
\text { reais }\end{array}$ \\
\hline $\begin{array}{l}\text { Otimização no } \\
\text { processo de } \\
\text { atendimento na central } \\
\text { de abastecimento } \\
\text { farmacêutico }\end{array}$ & Redução anual de 200 mil reais \\
\hline
\end{tabular}

4.3. Ganho de Capital Estrutural e Cultura Organi-

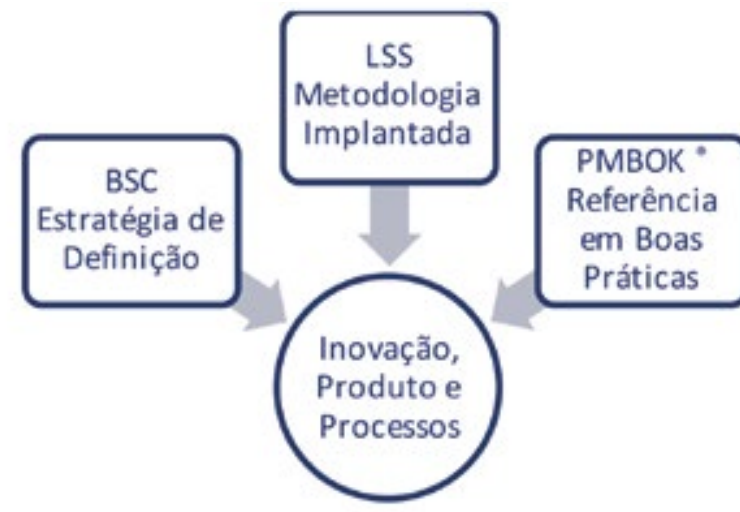

Figura 2. Estruturação da Gestão da Inovação

Para suportar a integração das três ferramentas foi escolhido um sistema de gestão de projetos que possibilitasse transparencia no acompanhamento e monitoramento dos projetos, garantindo o alinhamento deles às perspectivas do BSC, sustentado nas boas práticas do PMBOK®. Desde sua implementação, melhorias foram introduzidas, de forma contínua, atendendo a demandas institucionais geradas pelas informações disponibilizadas pelo próprio sistema. A mudança na cultura organizacional voltada a uma gestão efetiva dos projetos de inovação é um processo lento, mas de fundamental importância. Ela aparece como consequência das melhorias e funcionalidades implementadas, gerando benefícios para toda instituição hospitalar.

Esses projetos compartilham múltiplos stakeholders, com objetivos, compreensão e necessidades distintas, criando um desafio à gestão institucional da estrategia a ser adotada. Gerir esses projetos de maneira coesa e alinhada as iniciativas estrategicas do hospital é, em geral, um desafio conduzido de maneira pouco sistematizada neste nicho de mercado (terceiro setor). Isso contrasta com o potencial competitivo associado a gestão eficaz de um programa como este, desenvolvido pelo hospital escolhido para o estudo e evidenciando ganhos ainda no primeiro ano de implementação.

5. ANÁLISE PRELIMINAR QUALITATIVA DA ADERENCIA DA METODOLOGIA À GESTÃO DA INOVAÇÃO

A filosofia Lean Healthcare, absorvida a partir da implantação da metodologia LSS, associada a outras 
ferramentas de gestão, é importante para a saúde porque ela pode ajudar a criar soluções inovadoras, de alto impacto, sem necessariamente precisar
investir muito para isso acontecer. Por meio dessa investir muito para isso acontecer. Por meio dessa abordagem, e possivel catalisar soluções pontuais, bem como soluçoes mais holisticas para o sistema de saúde.

A definição de metas institucionais em uma implementação de Lean Seis Sigma deve ser desmembrada do nivel estratégico ate operacional e a melhor forma de conquistar este pleito e o espelhamento dos objetivos e diretrizes estratégicas nos projetos Lean Seis Sigma à luz das perspectivas do BSC, considerando as boas práticas de gerenciamento de projetos apontadas pelo $\mathrm{PMBOK}{ }^{\circledR}$.

Com este entendimento, já solidificado e consolidado pela instituição, é possível para a organização hospitalar ponderar em seu planejamento orçamentário métricas para neutralização da inflação, considerando a realização de projetos e metas por equipes

de Black e Green Belts

1. Utilzando o Total Cost do ano anterior, atribuin do um percentual de inflação de custo projetado do ano em curso, definindo uma meta global instituciona

2. Definido o pipeline de projetos por área, os times de Black/Green Belts deverão ser montados para estudar inovações de produtos e/ou serviços estratégicos.

3. Os Black/Green Belts utilizarão a ajuda dos Yellow Belts para implementar as melhorias dos processos e poderão medir em um ano os ganhos Deployment Champion.

O modelo de gestão de inovação adotado pelo hospital em estudo, tendo como base ferramentas de gestão sólidas e ágeis, evidenciou conquistas técn cas e administrativas, que agregam valor direto ao cliente paciente, cliente médico e o colaborador na condição de cliente interno.

Figura 3. Modelo de Gestão de Inovação de Produtos e Processos do Hospital

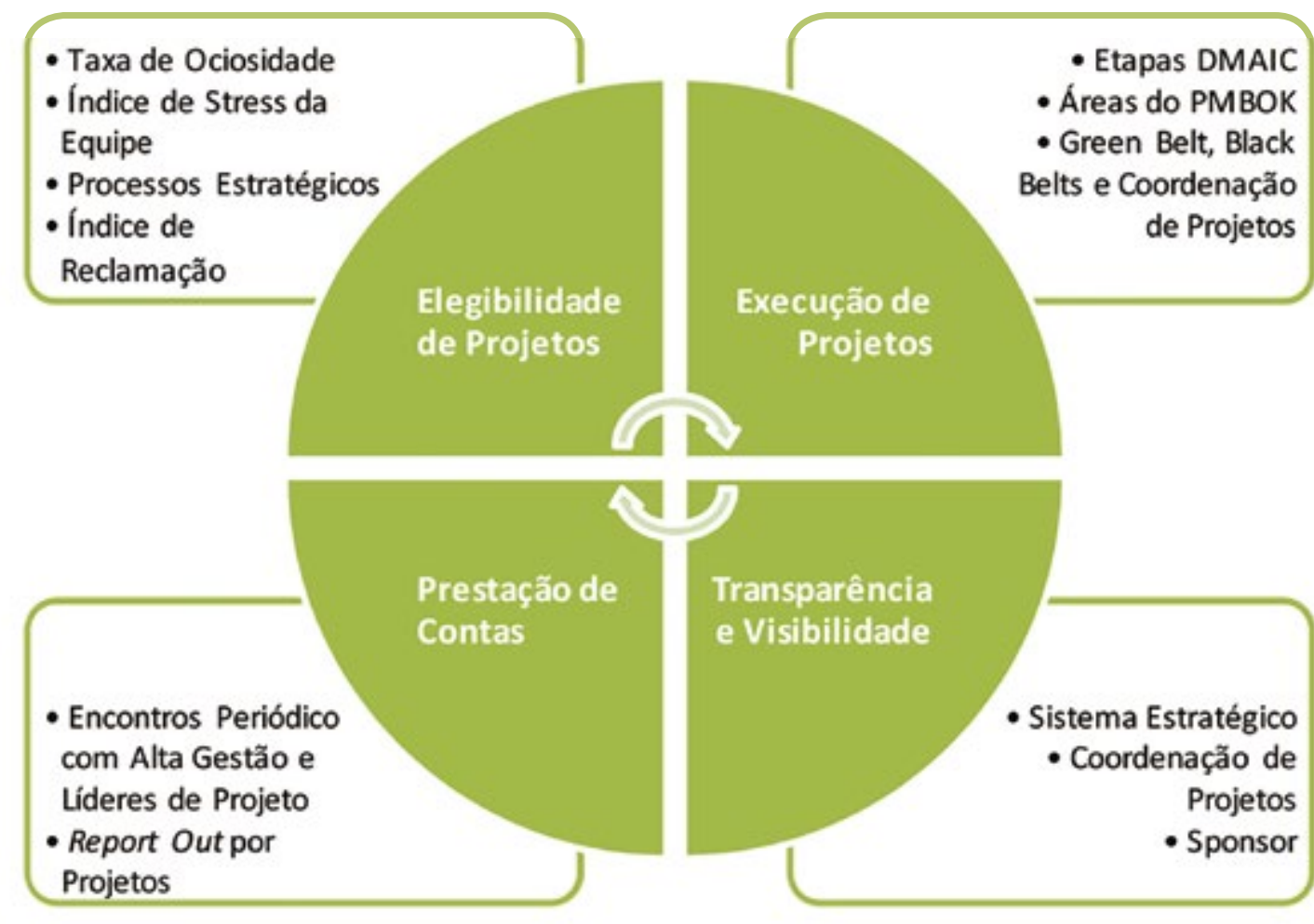

Como resultado, o envolvimento de diferentes proissionais, de diferentes áreas e papéis nas atividades de projetos, tem contribuído para uma cultura favorável para a inovação. Hoje, as instâncias de coordenação podem monitorar esses resultados por meio de indicadores de processos incorporados aos relatorios das àreas e da diretoria, avaliad Balanced Score Card (BSC).

\section{REFERÊNCIAS}

1. Keller P., Pyzdek T. Seis Sigma, Guia do Profissional - Um guia completo para Green Belts, Black Belts e Gerentes em Todos os Niveis.

2. Pereira JC, Saes SG, Escuder MM. Definindo prioridades de gestão de ciência e tecnologia em saúe. Rev. Saúde Pública. 1997;31(6):624-31

3. Rotondaro RG. Seis Sigma: estratégia gerencial para a melhoria de processos, produtos e serviços. São Paulo: Atlas; 2002.

4. Werkema MC. Lean Seis Sigma Introdugma: Estratégia gerencial manufacturing. Rio de Janeiro: ekema; 2006.

5. NAVE D. Como comparar o Seis Sigma, o Lean e a Teoria das Restrições. http://agente.epse. 6. 6. Valle AB, Soares CA, Finocchio Junior J, Silva LS. Fundamentos do gerenciamento de projetos. Rio de Janeiro: FGV Management; 2007.

7. Project Management Institute (PMI). Um guia do conhecimento em gerenciamento de projetos (Guia PMOK()). $4^{a}$ ed. Atlanta: Global Standard; 2008.

8. Kaplan RS, Norton DP. A Estratégia em Ação: Balanced Scorecard - Editora Campus, São Paulo, 1997.

9. Arbache AP. Metodologia científica para TCC São Paulo: Fundação Getúlio Vargas; 2010.

10. Marshall Junior I, Cierco AA, Rocha AV, Mota $\mathrm{EB}$, Leusin S. Gestão da qualidade. $10^{\mathrm{a}} \mathrm{ed}$. Rio de Janeiro: FGV; 2010

11. Prado D. Maturidade em gerenciamento de projetos. $2^{\mathrm{a}}$ ed. Novo Lima: INDG; 2010.

12. Fornari AR. Estudo comparativo da aderência de ferramentas livres ao PMBOK® (2004). Rio de Janeiro; 2009. Disponível em: http://www.bcc.ufla.br/ monografias/2010/Estudo_Comparativo_da_Aderencia_de_Ferramentas_Livres_ao_PMBOK.pdf

13. http://evolucaoagora.blogspot.com $\mathrm{br} / 2012 / 10 /$

14. http://www.fnq.org.br/informe-se/artigos-e-entrevistas/entrevistas/gestao-da-inovacao-nas-organizacoes
15. http://portal.blbbrasilescoladenegocios.com $\mathrm{br} / g$ estao-da-inovacao-nas-empresas/

16. hitps://www.dicio.com.br/

17. Henrique, D. B. Modelo de Mapeamento de Fluxo de Valor para Implantaçoes de Lean em Amc̃̃o ção de Mestrado em Engenharia de Produçáo, Untversidade de São Paulo; 2014

1- Coordenadora de Projetos do HS Endereço para correspondência: 